\title{
ИСХОДЫ ОСТРОГО ИНФАРКТА МИОКАРДА В ПЕРИОД ПАНДЕМИИ В 2020 ГОДУ И ДО НЕЕ В 2019 ГОДУ
}

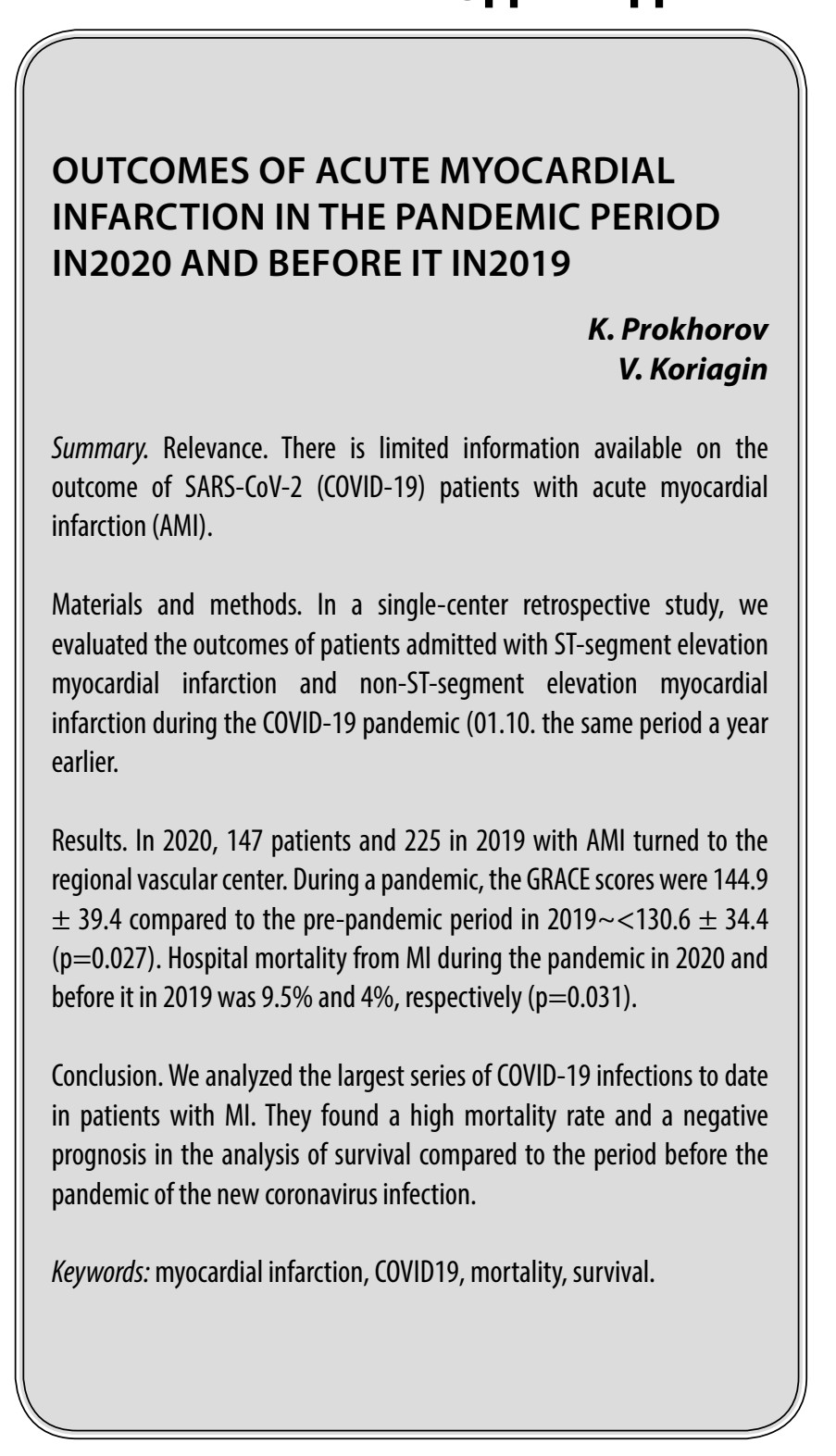

\section{Актуа^ьность}

H есмотря на широкое использование методов реперфузии и улучшение дополнительных медицинских методов лечения, пациенты с острым инфарктом миокарда (ОИМ) по-прежнему представляют значительный риск сердечно-сосудистых событий и смертности $[1,2]$. По этой причине были установлены несколько факторов риска для прогнозирования неблагоприятного развития в краткосрочной и долгосрочной перспективе [3].
Прохоров Кирилл Владимирович

Соискатель, Пермский государственный медицинский университет им. академика Е.А. Вагнера; главный врач ГБУЗ ПК ККД kirpro059@gmail.com

Корягин Владимир Сергеевич Пермский государственный медицинский университет им. академика Е.А. Вагнера vladimirkoryagin12@gmail.com

Аннотация. Актуальность. Имеется ограниченная информация об исходах пациентоB с заболеванием SARS-CoV-2 (COVID-19) с острым инфарктом миокарда (ОИМ).

Материалы и методы. В одноцентровом ретроспективном исследовании мы оценили исходы пациентов, поступивших с инфарктом миокарда с подъемом сегмента ST и инфарктом миокарда без подъема сегмента ST во время пандемии COVID-19 (01.10.2020-30.11.2020 г.) по сравнению с пациентами с ОИМ, поступившими в тот же период годом ранее.

Результаты. В 2020 году обратилось 147 пациентов и 225 в 2019 с ОИМ В региональный сосудистый центр. Во время пандемии баллы по шкале GRACE144,9 39,4 по сравнению с периодом до пандемии в 2019 году $130,6 \pm 34,4$ ( $p=0,027)$. Госпитальная летальность от ИМ в период пандемии в 2020 году и до нее в 2019 году составила 9,5\% и 4\%, соответственно $(p=0,031)$.

Заключение. Мы провели анализ крупной на сегодняшний день серии случаев инфицирования COVID-19 пациентов с ИМ. Обнаружили высокую летальность и отрицательный прогноз по анализу выживаемости по сравнению с периодом до пандемии новой коронавирусной инфекции.

Ключевые слова: инфаркт миокарда, COVID19, летальность, выживаемость.

\section{Материа^ и методы}

Ретроспективный одноцентровой обзор медицинских карт пациентов, последовательно госпитализированных в период с 01 октября 2020 г. по 30 ноября 2020 г. с COVID-19 и ИМ, был проведен в региональном сосудистом центре (РСЦ) Пермского края. В 2020 году обратилось 147 пациентов и 225 в 2019 с ОИМ. Статистический анализ проводился с использованием версии 25 пакета программного обеспечения IBM SPSS Statistics (IBM Corp., США). 
Таблица 1. Тяжесть острого инфаркта миокарда в период пандемии в 2020 году и до нее в 2019 году

\begin{tabular}{|c|c|c|c|c|c|}
\hline \multirow[t]{2}{*}{ Тяжесть ИМ } & \multicolumn{2}{|c|}{$\begin{array}{l}1 \text { группа } \\
\text { Пандемия } 2020 \\
(\mathrm{n}=147) \\
\end{array}$} & \multicolumn{2}{|c|}{$\begin{array}{l}2 \text { группа } \\
\text { До пандемии } 2019 \\
\text { (n=225) }\end{array}$} & \multirow[t]{2}{*}{$p$} \\
\hline & $\mathrm{M} \pm \mathrm{SD}$ & 95\% ДИ & $\mathrm{M} \pm \mathrm{SD}$ & 95\% ДИ & \\
\hline Баллы по шкале GRACE & $144,9 \pm 39,4$ & $126,05-135,14$ & $130,6 \pm 34,4$ & $126,05-135,14$ & $0,027^{*}$ \\
\hline
\end{tabular}

* - различия показателей статистически значимы $(p<0,05)$

Таблица 2. Госпитальная летальность от ИМ в период пандемии в 2020 году и до нее в 2019 году

\begin{tabular}{|c|c|c|c|c|c|c|}
\hline \multirow{3}{*}{ Летальность ИМ } & \multicolumn{4}{|c|}{ Распространенность по годам } & \multirow{3}{*}{$p$} & \multirow{3}{*}{ ОШ; 95\% ДИ } \\
\hline & \multicolumn{2}{|c|}{$\begin{array}{l}1 \text { группа } \\
\text { Пандемия } 2020 \\
(n=147) \\
\end{array}$} & \multicolumn{2}{|c|}{$\begin{array}{l}2 \text { группа } \\
\text { До пандемии } 2019 \\
(\mathrm{n}=225)\end{array}$} & & \\
\hline & $\begin{array}{l}\text { Абс., } \\
\text { чел }\end{array}$ & $\%$ & Абс., чел & $\%$ & & \\
\hline Всего & 14 & 9,5 & 9 & 4,0 & $0,031^{*}$ & 2,$526 ; 1,064-5,999$ \\
\hline
\end{tabular}

Таблица 3. Показатели смертности пациентов с ОИМ в период пандемии в 2020 году и до нее в 2019 году

\begin{tabular}{|c|c|c|c|}
\hline \multirow{2}{*}{ Группа } & \multirow{2}{*}{$\begin{array}{l}\text { Время с момента выписки, } \\
\text { дней }\end{array}$} & \multicolumn{2}{|c|}{ Риск смерти пациента } \\
\hline & & A6c. & $\%$ \\
\hline \multirow{7}{*}{$\begin{array}{l}1 \text { группа } \\
\text { Пандемия } 2020 \\
(n=8)\end{array}$} & 0 & 1 & 88,0 \\
\hline & 30 & 4 & 38,0 \\
\hline & 60 & 1 & 25,0 \\
\hline & 90 & 1 & 13,0 \\
\hline & 120 & 1 & 0,0 \\
\hline & 150 & 0 & 0,0 \\
\hline & 180 & 0 & 0,0 \\
\hline \multirow{7}{*}{$\begin{array}{l}2 \text { группа } \\
\text { До пандемии } 2019 \\
(n=17)\end{array}$} & 0 & 3 & 82,0 \\
\hline & 30 & 0 & 82,0 \\
\hline & 60 & 0 & 82,0 \\
\hline & 90 & 0 & 82,0 \\
\hline & 120 & 1 & 76,0 \\
\hline & 150 & 0 & 76,0 \\
\hline & 180 & 2 & 65,0 \\
\hline
\end{tabular}

\section{Результаты}

Крупный международный наблюдательный Глобальный регистр острых коронарных событий (GRACE) также продемонстрировал отличную способность оценивать риск смерти в стационаре [3]. Наиболее примечательным открытием нашего исследования является то, что пандемия НКВИ независимо связана с более высокой внутрибольничной смертностью у пациентов с ОИМ, также на показатель влияет и период изоляции на территории региона. В 2020 году пациенты были значимо тяжелее. Полученная

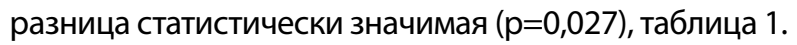

Имелось и увеличение летальности в региональном сосудистом центре Клинический кардиологический диспансер (таблица 2) среди пациентов, госпитализированных по поводу острого коронарного синдрома, проведено прямое сравнение показателей летальности 01.10.2020-30.11.2020 и 01.10.2019-30.11.2019, но сохраняется сложность напрямую оценить смертность и инвалидность, которые, вероятно, произошли среди тех пациентов с острым коронарным синдромом, которые не были госпитализированы из-за НКВИ и, следовательно, не получали лечение, которое, как известно, должно было быть эффективным. 


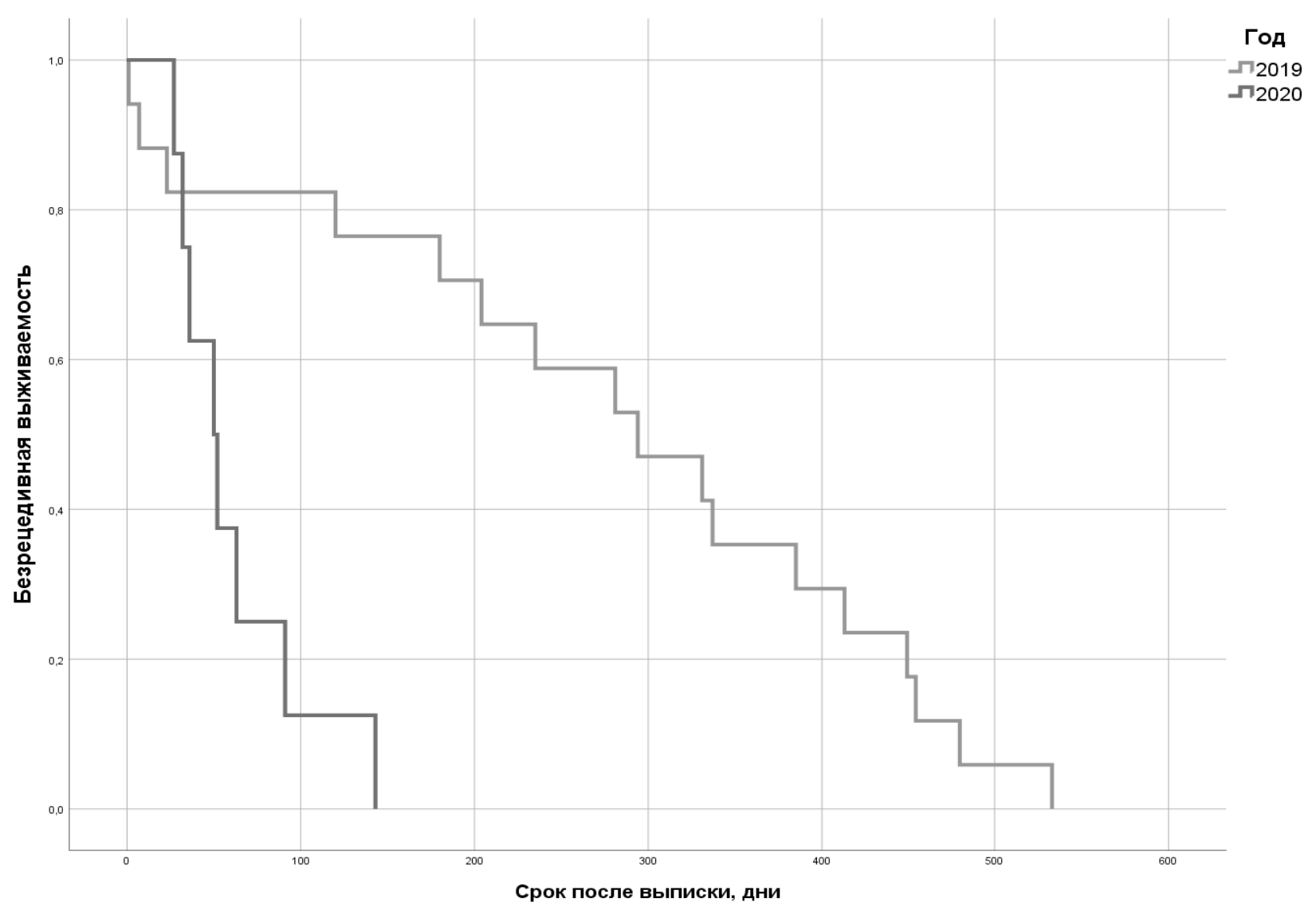

Рис. 1. Анализ безрецидивной выживаемости пациентов с острым инфарктом миокарда во время пандемии 2020 году и до нее в 2019 году

Летальность от ОИМ существенно увеличилась с 4\% до пандемии до 9,5\% (p=0,031) во время пандемии.

Тяжесть проявления ОИМ была более выраженной во время пандемии НКВИ [повышение уровней сердечных ферментов, увеличение потребности в инотропной поддержке на 25\% $(p<0,01)]$, в то время как демографические и ангиографические характеристики пациентов не различались между до пандемии и пандемии НКВИ.

Оценка зависимости вероятности смерти от дней после выписки была выполнена с помощью анализа выживаемости. Получение данные представлены в таблице дожития (таблица 3). В выборку попали только пациенты, которые умерли в течение 6 месяцев с момента выписки.

Анализ безрецидивной выживаемости пациентов, был выполнен с помощью метода кривых Каплана-Мейера (таблица 3).

В соответствии с полученными данными, среднее количество дней после выписки до смерти у пациентов 1 груп-

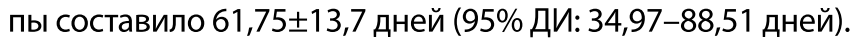

Медиана срока наблюдения во время пандемии (срок, в течение которого не менее чем у 50\% исследуемых отмечалась смерть) составила $50 \pm 11$ дней (95\% ДИ: 27,82-72,17 дней). Во второй группе среднее количество дней до смерти после выписки составило 278,05 $\pm 41,1$ дней (95\% ДИ: 197,51-358,61 дней), рисунок 1. Медиа-

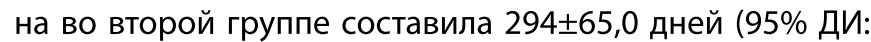
164,85-423,1 дней), рисунок 1.

\section{Обсужление}

На сегодняшний день данные о проявлениях, лечении и исходах ОИМ во время пандемии COVID-19 ограничены. Начало социального сдерживания - состояние изоляции - для уменьшения распространения инфекции COVID-19 было связано с почти 50\% снижением количества госпитализаций с ОИМ и значительно более высокой ранней смертностью от ОИМ при самом низшем уровне госпитализаций. Учитывая количество пациентов с ОИМ, которые не обращаются в больницу (и задержки в обращении среди госпитализированных) [4], в ближайшем будущем вероятно увеличение смертности от ОИМ среди населения на дому и увеличение 
количества госпитализаций по поводу сердечной недостаточности.

Во время второй волны пандемии в 2020 году имелось явление, связанное с отсроченным лечением ОИМ или его отсутствие что привело к увеличению смертности, связанных с ОИМ. Было выявлено, что пациенты, не страдающие COVID-19, не обращаются за госпитализацией из-за опасений по поводу риска внутрибольничной инфекции COVID-19, а также социальных ограничений, установленных правительствами и местными органами здравоохранения для борьбы с пандемией $[1,4]$.

Наше исследование предполагает, что эффективные организационные изменения могут противостоять неблагоприятным последствиям пандемии с точки зрения сдерживания внутрибольничной летальности для пациентов с ОИМ, обеспечивая надлежащий уровень клинической помощи госпитализированным пациентам, не страдающим заболеванием COVID-19. Более того, как было предложено в недавних исследованиях [5-7], органы общественного здравоохранения должны внедрять более эффективные коммуникационные стратегии во время эпидемии, чтобы обеспечить своевременное обращение к людям (предотвращение огромного увеличения времени до двери, зарегистрированного во время вспышки COVID-19) с острыми неинфекционными катастрофами, такие как ОИМ и инсульт.

По результатам нашего исследования выявлено, что половина погибших пациентов с ОИМ во время пандемии имели срок дожития всего 50 дней по сравнению с пациентами ОИМ в 2019 году. Эти данные требуют усиленного контроля на уровне поликлиники пациентов крайне высокого риска в течение первых двух месяцев после сосудистой катастрофы.

\section{ЛИТЕРАТУРА}

1. M. Piironen, 0. Ukkola, H. Huikuri, et al. Trends in long-term prognosis after acute coronary syndrome. Eur J Prev Cardiol., 24 (2017), pp. $274-280$.

2. P. Tobbia, B.R. Brodie, B. Witzenbichler, et al. Adverse event rates following primary PCI for STEMI at US and non-US hospitals: three-year analysis from the HORIZONSAMI trial. Eurolntervention., 8 (2013), pp. 1134-1142.

3. K.A. Eagle, M.J. Lim, 0.H. Dabbous, et al. A validated prediction model for all forms of acute coronary syndrome: estimating the risk of 6-month postdischarge death in an international registry. JAMA., 291 (2004), pp. 2727-2733.

4. Задержка оказания медицинской помощи пациентам с острым инфарктом миокарда во время пандемии COVID-19 / K.B. Прохоров, H.A. Корягина, Г.Н. Спасенков [и др.] // Современная наука: актуальные проблемы теории и практики. Серия: Естественные и технические науки.— 2021.— № 8.— C. 202-205. - DOl 10.37882/2223-2966.2021.08.29.

5. Zorzi A, Vio R, Rivezzi F. et al. Characteristics and hospital course of patients admitted for acute cardiovascular diseases during the coronavirus disease-19 outbreak. J Cardiovasc. Med. 2021: 22:29-35. pmid:33186239.

6. Nacoti M, Ciocca A, Giupponi A, et al. At the epicenter of the Covid- 19 pandemic and humanitarian crises in Italy: changing perspectives on preparation and mitigation. NEJM Catal. 2020.

7. Dunlop C, Howe A, Allen LN. The coronavirus outbreak: the central role of primary care in emergency preparedness and response. BJGP 0pen 2020; 1;4(1): bigopen20X101041. pmid:31992543

(с) Прохоров Кирилл Владимирович ( kirpro059@gmail.com ), Корягин Владимир Сергеевич ( vladimirkoryagin12@gmail.com ). Журнал «Современная наука: актуальные проблемы теории и практики» 